DOI:10.2478/rrlm-2021-0008

\title{
Predictive Value of Neutrophil Count for Postoperative Complications in Children after Surgery of Perforated Appendicitis
}

\author{
Vesna Marjanovic ${ }^{1,2^{*}}$, Ivana Budic ${ }^{1,2}$, Ljubinka Jankovic-Velickovic ${ }^{3,4}$, \\ Marija Stevic ${ }^{5,6}$, Milos Kostic ${ }^{7}$, Dusica Simic ${ }^{5,8}$
}

1. Department of Anesthesiology and Reanimatology, Faculty of Medicine, University of Nis, Serbia

2. Clinic for Anesthesia and Intensive Therapy, Clinical Centre Nis, Serbia

3. Department of Pathology, Faculty of Medicine, University of Nis, Serbia

4. Centre of Pathology, Clinical Centre Nis, Serbia

5. Department of Surgery with Anesthesiology, Faculty of Medicine, University of Belgrade, Serbia

6. Department of Anesthesiology, Reanimatology and Intensive Therapy, University Children's Hospital, Belgrade, Serbia

7. Department of Immunology, Faculty of Medicine, University of Nis, Serbia

8. Department of Anesthesiology, Reanimatology and Intensive Therapy, University Children's

Hospital, Serbia

\begin{abstract}
Introduction: Perforated appendicitis (PA) in children is associated with a considerable risk for postoperative complications (POCs) such as wound infection and intra-abdominal abscess. The aim of this study was to determine the diagnostic accuracy of hematological parameters in the early POC detection in children after PA surgery. Materials and Methods: The study enrolled 71 patients with PA divided into two groups: 14 patients with POC (POC+ group) and 57 patients without POC (POC-group). Clinical and hematological parameters were followed preoperatively, prior to the surgery (PRO) and postoperatively on day 2 (POD2) and day 4 (POD4). Results: The $P O C+$ group had longer duration of higher axillar temperature as well as extended intensive and inpatient care. This group also had a significantly lower absolute neutrophil count ratio between POD2 and POD4. According to the receiver operating characteristic curve analysis, relative neutrophil count on POD4 higher than $71.8 \%$ and the ratio of absolute neutrophil count between POD2 and POD4 lower than 44.5\% were found to be useful for predicting POC. Conclusion: Absolute neutrophil count ratio between POD2 and POD4 and relative neutrophil count at POD4 could be efficient in identifying children at higher risk of developing POC after PA surgery.
\end{abstract}

\footnotetext{
* Corresponding author: Vesna G. Marjanovic, Department of Anesthesiology and Reanimatology, Faculty of Medicine, University of Nis, Serbia. E-mail: drvesnamarjanovic@gmail.com
} 
Keywords: perforated appendicitis, predictors of postoperative complication, children

Received: 22 $2^{\text {nd }}$ October 2020; Accepted: 29 th December 2020; Published: $14^{\text {th }}$ January 2021

\section{Introduction}

Perforation complicates $32.5 \%$ of appendicitises in children (1). Perforated appendicitis (PA) represents a surgical emergency requiring immediate appendicectomy. In this setting, postoperative complications (POCs) occur in 6.4-13.2\% of cases (2). Post appendicectomy complications in children including abdominal abscess, wound infection, ileus and mechanical intestinal obstruction, result in prolonged hospital stay, increased morbidity and higher treatment cost $(2,3)$. Thus, predicting complications after PA surgery are important both for the patient and the healthcare service.

The most frequently used laboratory parameters in POC identification after pediatric appendicectomy are white blood cell count (WBC), C-reactive protein (CRP) level (4) and lymphocyte count (5). Until now, these laboratory parameters were considered auxiliary alongside positive ultrasonographic POC signs, considering that no single hematological parameter or their ratio appears to be reliable in identifying patients at higher risk for POC development. We hypothesized that during the postoperative period, the degree of inflammatory process as a consequence of an emerging infection due to POCs, would correlate with hematological parameters alone and/or with their ratio.

Therefore, the aim of this study was to determine the postoperative diagnostic accuracy of various hematological parameters in the identification of children prone to develop POCs after PA surgery.

\section{Materials and Methods}

This retrospective study included 71 pediatric patients (46 males, 25 female), aged between 3-17 years, who underwent emergency appendi- cectomy for PA from January 2015 until January 2019 at the Department of Pediatric Surgery, Clinical Centre Nis. The study was approved by the Ethics Committee of the Clinical Centre Nis (No.7202/2019). This body waived the need for written informed consent of the patients as the study was considered an audit of routine practice.

Appendicitis diagnosis was based on clinical presentation, laboratory and imaging results (abdominal radiography and sonography). Emergency appendicectomies were all performed as open surgical procedures. Patients with gangrenous, non-perforated, appendicitis were not included in the study. The diagnosis of PA was confirmed intraoperatively (6) and by pathohistological examination. Systemic antibiotic prophylaxis was performed with ceftriaxone and metronidazole in accordance with the local hospital guidelines.

All study participants were classified into two groups: with (POC+) and without (POC-) postoperative complications. Postoperative complications were defined as a wound infection and intra-abdominal abscess developing within the 30 days after surgery. Centers for Disease Control and Prevention criteria were used to define wound infection. Any liquid collection, detected by abdominal radiography and ultrasound imaging during the postoperative period was considered to be intra-abdominal abscess (7). Other POCs were not considered for this study.

The patients' data including demographic characteristics, duration of pre-hospital symptoms, hospital observations (from admission until the time of operation), operation time, POC presence, duration and the degree of axillary temperature in preoperative and postoperative peri- 
od, duration of Intensive Care Unit (ICU) stay and overall hospital stay were retrieved from the medical records.

Hematological parameters, including WBC, relative lymphocyte (Ly\%) and neutrophil $(\mathrm{Ne} \%)$ count, absolute count of lymphocytes (ACL) and neutrophils $(\mathrm{ACN})$ were measured prior to surgery (PRO) and on postoperative day 2 (POD2) and 4 (POD4). The ratio of WBC, ACL and ACN was calculated between PRO/POD2, POD2/ POD4 and PRO/POD4 time points. The higher axillary temperature was defined as a temperature higher than $37^{\circ} \mathrm{C}$.

The cohort and the investigated parameters were characterized using descriptive statistics. Frequency of attributive variables between groups was compared using the Pearson $\chi^{2}$ test and Fisher's exact test. The comparison of means between groups and non-parametric POC variables was done using the t-test and Mann-Whitney test, respectively. The receiver operating characteristic (ROC) curve analysis was used to determine the optimal cut off values for investigated hematological parameters. The performance of each parameter in POC prediction was compared by calculating the area under the curve (AUC). Statistical analysis was done using Microsoft Office Excel and R-software. The value of $p<0.05$ was considered statistically significant.

\section{Results}

Of 71 enrolled patients, 14 (19.72\%) developed POCs: $3(4.23 \%)$ had wound infection and 11 (15.49\%) had a postoperative abscess. The median time of POC emergence was 7 days after surgery (ranging 5-12 days).

There were no significant differences in the age, gender, body weight, duration of pre-hospital symptoms, hospital observation time and operation time between the POC+ and POC-group. Patients with POCs had longer periods of pyrexia as well as higher degree of axillary temperature on POD2 and POD4. Also, the POC+ group had a longer ICU and overall hospital stay (Table 1). Among investigated hematological parameters, $\mathrm{WBC}, \mathrm{Ne} \%$ and $\mathrm{ACN}$ were found to be higher, whereas $\mathrm{Ly} \%$ and ACL lower in $\mathrm{POC}+$ patients, however, the observed differences did not reach statistical significance. On the other hand, the ratio between $\mathrm{ACN}$ measured on POD2 and

Table 1. Demographic and clinical characteristics of study participants

\begin{tabular}{lccc}
\hline & POC- group & POC+ group & p value \\
\hline Number of patients (\%) & $57(80.28)$ & $14(19.72)$ & \\
Age (years) & $9.77 \pm 4.05$ & $8.71 \pm 4.38$ & 0.399 \\
Male sex (\%) & $37(64.9)$ & $9(64.3)$ & 0.590 \\
Body weight (kg) & $38.66 \pm 18.75$ & $33.86 \pm 19.61$ & 0.293 \\
Duration of pre-hospital sym. (h) & $37.53 \pm 29.70$ & $54.07 \pm 33.73$ & 0.092 \\
Hospital observation (h) & $7.81 \pm 7.53$ & $10.75 \pm 9.19$ & 0.373 \\
Operation time (h) & $1.20 \pm 0.44$ & $1.30 \pm 0.38$ & 0.196 \\
Duration of higher axil. temp. at hospital (days) & $2.26 \pm 1.34$ & $5.93 \pm 4.36$ & $<0.001^{*}$ \\
Axil. Temp $\left({ }^{\circ} \mathrm{C}\right)$ at: & & & \\
PRO & $37.94 \pm 0.86$ & $37.92 \pm 0.88$ & 0.932 \\
POD2 & $37.21 \pm 0.52$ & $37.54 \pm 0.50$ & $<0.05^{*}$ \\
POD4 & $36.87 \pm 0.41$ & $37.33 \pm 0.40$ & $<0.001^{*}$ \\
ICU stay (days) & $3.62 \pm 1.66$ & $6.77 \pm 5.57$ & $<0.005^{*}$ \\
Hospital stay (days) & $6.33 \pm 2.36$ & $14.21 \pm 3.51$ & $<0.001^{*}$ \\
\hline
\end{tabular}

POC- group- patients without postoperative complications; POC + group- patients with postoperative complications; PRO- preoperatively, prior to surgery; POD2- postoperative day 2; POD4-postoperative day 4; ICU- Intensive Care Unit. 
POD4 was significantly lower in POC + group, (Table 2).

According to the ROC curve analysis, relative neutrophil count on POD4 and POD2/POD4 $\mathrm{ACN}$ ratio were the most accurate hematological markers in distinguishing the $\mathrm{POC}+$ and POCgroup (Table 3).

Utilizing the value of $\mathrm{Ne} \%$ on POD4 higher than $71.8 \%$ as a predictor of POC + group, the cut off point had a sensitivity of $55.6 \%$ and specifici-

Table 2. Hematological profile of patients with perforated appendicitis

\begin{tabular}{|c|c|c|c|}
\hline & POC-group & POC+ group & p value \\
\hline \multicolumn{4}{|l|}{ WBC $\left(\times 10^{9} / \mathrm{L}\right)$ at } \\
\hline PRO & $19.04 \pm 6.57$ & $17.74 \pm 5.38$ & 0.613 \\
\hline POD2 & $12.17 \pm 5.46$ & $13.12 \pm 5.93$ & 0.746 \\
\hline POD4 & $9.90 \pm 3.90$ & $11.56 \pm 7.83$ & 0.719 \\
\hline \multicolumn{4}{|l|}{$\mathrm{Ly} \%$ at } \\
\hline PRO & $11.24 \pm 5.97$ & $14.08 \pm 8.36$ & 0.269 \\
\hline POD2 & $15.73 \pm 7.59$ & $14.76 \pm 6.19$ & 0.800 \\
\hline POD4 & $25.00 \pm 7.55$ & $23.49 \pm 7.94$ & 0.415 \\
\hline \multicolumn{4}{|l|}{$\mathrm{Ne} \%$ at } \\
\hline PRO & $80.75 \pm 7.18$ & $80.86 \pm 8.50$ & 0.960 \\
\hline POD2 & $75.38 \pm 8.19$ & $79.11 \pm 7.59$ & 0.169 \\
\hline $\begin{array}{l}\text { PODZ } \\
\text { POD4 }\end{array}$ & $63.66 \pm 8.00$ & $68.56 \pm 7.43$ & 0.075 \\
\hline $\begin{array}{c}\operatorname{ACL}\left(\mathrm{x} 10^{9} / \mathrm{L}\right) \text { at } \\
\text { PRO }\end{array}$ & $1.90 \pm 0.78$ & $2.29 \pm 1.18$ & 0.263 \\
\hline POD2 & $1.74 \pm 0.78$ & $1.78 \pm 0.75$ & 0.753 \\
\hline POD4 & $2.39 \pm 1.05$ & $2.60 \pm 1.77$ & 0.783 \\
\hline \multicolumn{4}{|l|}{$\mathrm{ACN}\left(\mathrm{x} 10^{9} / \mathrm{L}\right)$ at } \\
\hline PRO & $15.42 \pm 5.98$ & $14.01 \pm 4.50$ & 0.414 \\
\hline POD2 & $9.31 \pm 4.60$ & $10.58 \pm 5.49$ & 0.570 \\
\hline POD4 & $6.33 \pm 2.67$ & $8.16 \pm 6.12$ & 0.443 \\
\hline \multicolumn{4}{|l|}{ WBC ratio at } \\
\hline PRO/POD2 & $1.71 \pm 0.70$ & $1.45 \pm 0.53$ & 0.358 \\
\hline POD2/POD4 & $1.26 \pm 0.43$ & $1.03 \pm 0.32$ & 0.131 \\
\hline PRO/POD4 & $2.02 \pm 0.79$ & $1.70 \pm 0.71$ & 0.274 \\
\hline \multicolumn{4}{|l|}{ ACL ratio at } \\
\hline PRO/POD2 & $1.21 \pm 0.55$ & $1.32 \pm 0.38$ & 0.263 \\
\hline POD2/POD4 & $0.70 \pm 0.25$ & $0.77 \pm 0.32$ & 0.603 \\
\hline PRO/POD4 & $0.85 \pm 0.39$ & $0.99 \pm 0.57$ & 0.645 \\
\hline \multicolumn{4}{|l|}{$\mathrm{ACN}$ ratio at } \\
\hline PRO/POD2 & $1.90 \pm 1.02$ & $1.51 \pm 0.65$ & 0.369 \\
\hline POD2/POD4 & $1.55 \pm 0.55$ & $1.15 \pm 0.39$ & $0.043^{*}$ \\
\hline PRO/POD4 & $2.62 \pm 1.27$ & $2.01 \pm 0.98$ & 0.173 \\
\hline
\end{tabular}

POC- group- patients without postoperative complications; POC+ group- patients with postoperative complications; PROpreoperatively, prior to surgery; POD2- postoperative day 2; POD4-postoperative day 4; WBC- white blood cell count; Ly\%relative count of lymphocytes; $\mathrm{Ne} \%$ - relative count of neutrophils; ACL- absolute count of lymphocytes, ACN- absolute count of neutrophils; PRO/POD2-between preoperative and postoperative day 2; POD2/POD4- between postoperative day 2 and 4; PRO/POD4- between preoperative and postoperative day 4 . 
Table 3. Receiver operating characteristics (ROC) curve analysis: ability of certain hematological parametres to differentiate patients at higher risk for postoperative complication

\begin{tabular}{lcccccc}
\hline & AUC & p & Cut off & Sensitivity (\%) & Specificity (\%) & 95\% CI \\
\hline $\mathrm{Ne} \%$ on POD4 & 0.756 & 0.02 & $71.8(\%)$ & $55.6 \%$ & $91.7 \%$ & $0.572-0.940$ \\
$\mathrm{ACN}$ ratio at POD2/POD4 & 0.264 & 0.03 & $44.5(\%)$ & $100 \%$ & $2.8 \%$ & $0.074-0.454$ \\
\hline
\end{tabular}

$\mathrm{Ne} \%$ - relative count of neutrophils; ACN ratio at POD2/POD4 - absolute neutrophil count ratio between postoperative day 2 and 4; AUC- Area under the curve; CI- confidence interval.

ty of $91.7 \%$. The POD2/POD4 ACN ratio lower than $44.5 \%$ had a sensitivity of $100 \%$ and specificity of $2.8 \%$ for determining patients with POC (Figure 1).

\section{Discussion}

The postoperative complications are still a major cause of morbidity, prolonged ICU staying and hospital treatment after appendectomy in children. The early identification of patients with POCs is crucial for timely and optimal treatment. Our data suggest that during postoperative period, $\mathrm{ACN}$ ratio and $\mathrm{Ne} \%$ could be useful diagnostic parameters.

Severe complications after PA surgery result in considerable morbidity and prolonged hospital stay, despite advances in diagnosis and the use of new, more potent antibiotics (3). The percentage of POCs differs between studies, ranging from 3 to $21 \%$, depending on defining criteria $(8,9)$. They are more frequent in patients with complicated appendicitis $(10,11)$, and in those managed by open appendicectomy (11). These adverse prognostic criteria were the basis for enrolment in our study. Our POC rate of $19.72 \%$ is in agreement with the results of other studies $(11,12)$. Some studies also reported that axillar temperature higher than $38^{\circ} \mathrm{C}$ on POD3 is associated with POC development, particularly postoperative abscesses $(13,14)$. Our results reiterate these findings, albeit the increase of axillary temperature may be lower than $38^{\circ} \mathrm{C}$ on POD2 and POD4. As previously reported, POC+ patients also had prolonged ICU and overall hospital stay $(3,15)$.
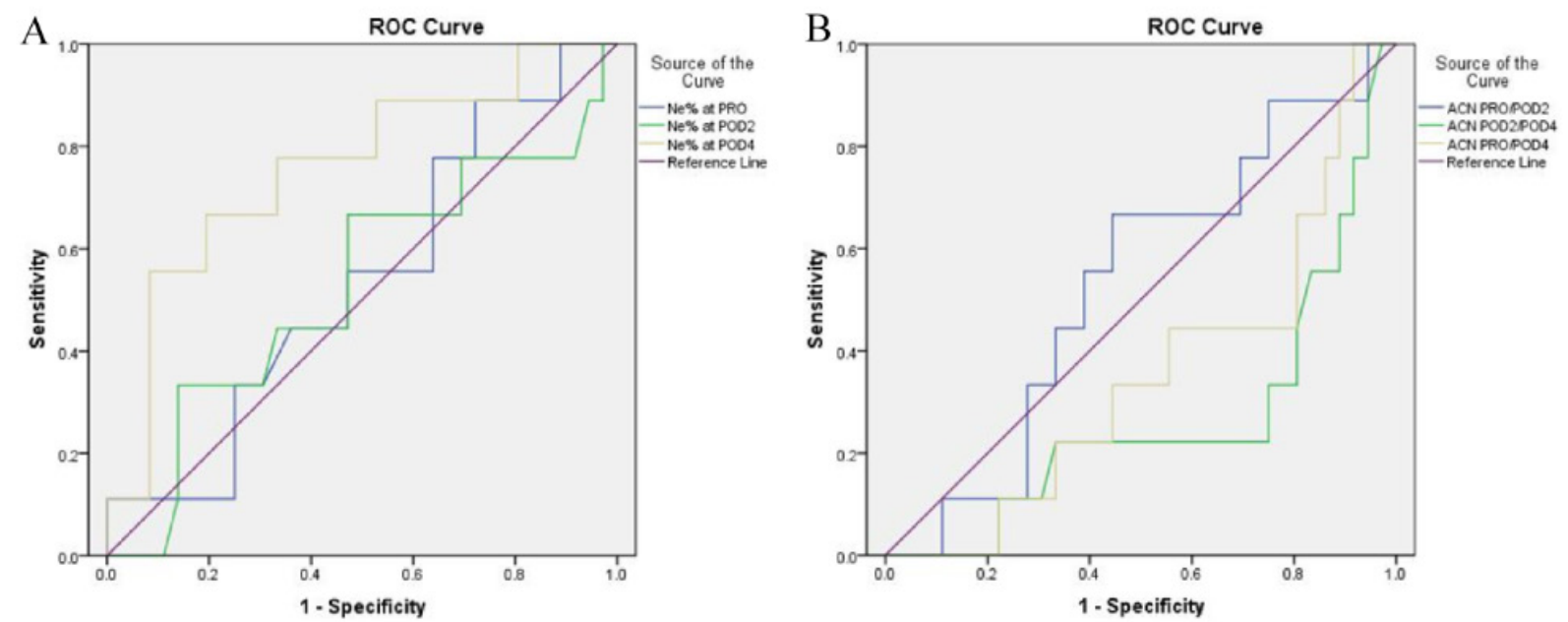

Fig. 1. Receiver operating characteristics (ROC) curves for relative count of neutrophils (A) and absolute neutrophil count ratio at different time intervals (B) 
The identification of patients at risk for POCs would be of clinical importance, especially in the view of an early diagnosis. Some authors reported that preoperative increase of $\mathrm{WBC}$ and CRP $(4,16,17)$ and the decrease of lymphocytes (5), could point towards POC development. However, other studies report that a preoperative increase of these parameters is the consequence of developed peritonitis in the context of complicated appendicitis and could not be used as reliable POC predictors (18). Several laboratory parameters were used to identify inflammatory activity during the postoperative period. Namely, postoperative increase of WBC on postoperative day 5 (19) and decrease of lymphocytes (5), might be signs of a postoperative abscess after appendectomy, however, only decrease of lymphocytes had predictive value, at the time of ultrasound confirmation. The question arises as to whether haematological parameters alone or their ratio in the postoperative period can be useful in POC detection before ultrasound confirmation. We found that lower ACN ratio between POD2 and POD4 $(<44.5 \%)$ and higher $\mathrm{Ne} \%$ on POD4 $(>71.8 \%)$, were useful parameters in discriminating pediatric patients with POCs after PA surgery. These results could be explained as a consequence of an emerging infection. Bratton et al. (20) reported that neutrophil count normalization occurs between 48-72 hours after resolution of infection, which is in agreement with our results. Until recently ACN was used only for the diagnosis and differentiation between simple appendicitis and PA (21). We found that ACN determined for each postoperative day is a worse POC predictor than POD2/POD4 ACN ratio, which provides a better insight into inflammatory events during the postoperative course. This parameter would greatly help in POC diagnosis before ultrasound confirmation, which is also suggested by others (22).

There are limitations to this study. The retrospective design of medical records and small number of patients with POCs may be a limiting factor and a weak point of our study.

\section{Conclusion}

Children with POC after PA surgery have lower POD2/POD4 ACN ratio and higher $\mathrm{Ne} \%$ at POD4 in comparison to uncomplicated cases, indicating that close monitoring of these parameters could facilitate the early identification of cases likely to develop POCs. These findings are of practical importance, considering that investigated parameters do not require any additional time or cost and are routinely performed in clinical practice. Taking into consideration that CRP and procalcitonin are important inflammatory parameters in everyday clinical practice of pediatric patients after PA surgery, comparison of these parameters with ACN ratio may be useful in future studies.

\section{Abbreviations}

ACL: absolute count of lymphocytes

ACN: absolute count of neutrophils

AUC: area under the curve

CRP: $C$ reactive protein

CI: confidence interval

ICU: Intensive Care Unit

Ly\%: relative lymphocyte count

$\mathrm{Ne} \%$ : relative neutrophil count

PA: perforated appendicitis

POC: postoperative complication

PRO: preoperatively

POD2: postoperative day 2

POD4: postoperative day 4

ROC: receiver operating characteristic

WBC: white blood cell count

\section{Acknowledgment}

This paper was supported by Internal project of The Medical Faculty University of Nis, No:42. 


\section{Authors' Contributions}

MV: conceptualization, methodology, investigation, formal analysis, writing of original draft; $\mathrm{BI}$ : investigation, writing review, editing, $\mathrm{J}-\mathrm{V}$ Lj: formal analysis, writing review, editing; SM: writing review, editing;

KM: formal analysis, writing review, editing; DS: writing review, editing.

\section{Conflicts of Interest}

The authors declare no conflict of interest.

\section{References}

1. Smink DS, Fishman SJ, Kleinman K, Finkelstein JA. Effects of race, insurance status, and hospital volume on perforated appendicitis in children. Pediatrics. 2005;115:920-5. DOI: 10.1542/peds.2004-1363

2. Kokoska ER, Silen ML, Tracy TF, Dillon PA, Cradock TV, Weber TR. Perforated appendicitis in children: risk factors for the development of complications. Surgery. 1998;124:619-25. DOI: 10.1067/msy.1998.91484

3. Fike FB, Mortellaro VE, Juang D, Sharp SW, Ostlie DJ, St Peter SD. The impact of postoperative abscess formation in perforated appendicitis. J Surg Res. 2011;170:24-6. DOI: 10.1016/j.jss.2011.03.038

4. Obayashi J, Ohyama K, Manabe S, Tanaka K, Nagae $\mathrm{H}$, Shima $\mathrm{H}$, et al: Are there reliable indicators predicting post-operative complications in acute appendicitis? Pediatr Surg Int. 2015;31:1189-1193. DOI: 10.1007/ s00383-015-3786-9

5. Lodwick DL, Cooper JN, Kenney B, Deans KJ, Minneci PC, Thakkar RK. Lymphocyte depression as a predictor of postoperative intraabdominal abscess after appendectomy in children. J Pediatr Surg. 2017;52:937. DOI: $10.1016 /$ j.jpedsurg.2016.10.028

6. St Peter SD, Sharp SW, Holcomb GW3rd, Ostlie DJ. An evidence based definition for perforated appendicitis derived from a prospective randomized trial. J Pediatr Surg. 2008;43:2242-5. DOI: 10.1016/j.jpedsurg.2008.08.051

7. Berríos-Torres SI, Umscheid CA, Bratzler DW, Leas B, Stone EC, Kelz RR, et al. Centers for Disease Control and Prevention Guideline for the Prevention of Surgical Site Infection. JAMA Surg. 2017;152:784-91. DOI: 10.1001/jamasurg.2017.0904
8. Lee SL, Ho HS. Acute appendicitis: is there a difference between children and adults? Am Surg. 2006;72:40913. DOI: $10.1177 / 000313480607200509$

9. Swank HA, Eshuis EJ, van Berge Henegouwen MI, Bemelman WA. Short- and long-term results of open versus laparoscopic appendectomy. World J Surg. 2011;35:1221-6. DOI: 10.1007/s00268-011-1088-5

10. Giraudo G, Baracchi F, Pellegrino L, Dal Corso HM, Borghi F. Prompt or delayed appendectomy? Influence of timing of surgery for acute appendicitis. Surg Today. 2013;43:392-6. DOI: 10.1007/s00595-012-0250-5

11. Tsioplis C, Brockschmidt C, Sander S, Henne-Bruns D, Kornmann M. Factors influencing the course of acute appendicitis in adults and children. Langenbecks Arch Surg. 2013;398:857-67. DOI: 10.1007/s00423-0131096-z

12. Katkhouda N, Mason RJ, Towfigh S, Gevorgyan A, Essani R. Laparoscopic versus open appendectomy: a prospective randomized double-blind study. Ann Surg. 2005;242:439-50. DOI: 10.1097/01. sla.0000179648.75373.2f

13. Giesen LJX, van den Boom AL, van Rossem CC, den Hoed PT, Wijnhoven BP. Retrospective Multicenter Study on Risk Factors for Surgical Site Infections after Appendectomy for Acute Appendicitis. Dig Surg. 2017;34:103-7. DOI: 10.1159/000447647

14. Fraser JD, Aguayo P, Sharp SW, Snyder CL, Holcomb GW 3rd, Ostlie DJ, et al. Physiologic predictors of postoperative abscess in children with perforated appendicitis: subset analysis from a prospective randomized trial. Surgery. 2010;147:729-32. DOI: 10.1016/j. surg.2009.10.057

15. Chen CL, Chao HC, Kong MS, Chen SY. Risk Factors for Prolonged Hospitalization in Pediatric Appendicitis Patients with Medical Treatment. Pediatr Neonatol. 2017;58:223-8. DOI: 10.1016/j.pedneo.2016.02.011

16. Shimizu T, Ishizuka M, Kubota K. The preoperative serum C-reactive protein level is a useful predictor of surgical site infections in patients undergoing appendectomy. Surg Today. 2015;45:1404-10. DOI: 10.1007/ s00595-014-1086-y

17. Shelton JA, Brown JJ, Young JA. Preoperative C-reactive protein predicts the severity and likelihood of complications following appendicectomy. Ann R Coll Surg Engl. 2014;96:369-72. DOI: 10.1308/003588414X139 46184901722

18. Gorter RR, van den Boom AL, Heij HA, Kneepkens 
FCM, Hulsker CC, Tenhagen M, et al. A scoring system to predict the severity of appendicitis in children. J Surg Res. 2016;200:452-9. DOI: 10.1016/j.jss.2015.08.042

19. Dickinson CM, Coppersmith NA, Luks FI. Early Predictors of Abscess Development after Perforated Pediatric Appendicitis. Surg Infect (Larchmt). 2017;18:8869. DOI: 10.1089/sur.2017.134

20. Bratton DL, Henson PM. Neutrophil Clearance: when the party's over, clean-up begins. Trends Immunol. 2011;32(8):350-7. DOI: 10.1016/j.it.2011.04.009
21. Bonadio W, Shahid S, Vardi L, Buckingham C, Kornblatt A, Free C, et al. A pre-operative clinical scoring system to distinguish perforation risk with pediatric appendicitis. J Pediatr Surg. 2018;53(3):441-5. DOI: 10.1016/j.jpedsurg.2017.05.017

22. Emil S, Elkady S, Shbat L, Youssef F, Baird R, Laberge $\mathrm{JM}$, et al. Determinants of postoperative abscess occurrence and percutaneous drainage in children with perforated appendicitis. Pediatr Surg Int. 2014;30(12):126571. DOI: $10.1007 / \mathrm{s} 00383-014-3617-4$ 